\title{
Interfacial and Aggregation Behaviour of Sodium Dodecyl Sulphate Induced by lonic Liquids
}

\author{
Manas Kumar Mandal', Manas Barai ${ }^{1}$, Habiba Sultana1, Emili Manna', Dulal Musib², \\ Dilip Kumar Maiti ${ }^{3}$, and Amiya Kumar Panda ${ }^{1 *}$ \\ ${ }^{1}$ Department of Chemistry, Vidyasagar University, Midnapore-721102, West Bengal, INDIA \\ ${ }^{2}$ Department of Chemistry, National Institute of Technology Manipur, Langol, Imphal-795004, INDIA \\ ${ }^{3}$ Department of Chemistry, University of Calcutta, 92 A.P.C. Road, Kolkata-700009, West Bengal, INDIA
}

\begin{abstract}
Aggregation studies of anionic surfactant sodium dodecyl sulphate (SDS) was investigated in aqueous 1-butyl-3-methylimidazolium chloride [bmim]Cl and $N$-butyl- $N$-methyl pyrrolidinium tetrafluoroborate $[\mathrm{bmp}] \mathrm{BF}_{4}$ ionic liquid (IL) solutions respectively. Systems were studied by surface tension, conductance, UV-VIS absorption/emission spectroscopy and dynamic light scattering. Critical micelle concentration $(C M C)$ values gradually decreased with increasing IL concentration which indicates synergistic interaction between ILs and SDS. Gibbs free energy change results demonstrated spontaneous micellization induced by ILs; however the effect of ILs were not similar to the corresponding regular salts $\left(\mathrm{NaCl}\right.$ and $\left.\mathrm{NaBF}_{4}\right)$. Aggregation number (n) of micelles, determined by fluorescence quenching method, indicate that the ' $n$ ' values increase with increasing ILs concentration, induced by the oppositely charged IL cation. Size of the micelles, determined by dynamic light scattering studies, increased with increasing ILs concentration, which were due to the formation of larger aggregates; the aggregates are considered to be comprised of the anionic surfactant with a substantial proportion of ILs cation as the bound counter ions. Such studies are considered to shed further light in the fundamentals of IL induced micellization as well as in different practical applications.
\end{abstract}

Key words: ionic liquid, surfactant, SDS, micelle, aggregation, spectroscopy

\section{Introduction}

Surfactants exhibit amphiphilic natures that alter the surface tension at air-liquid interface. Self aggregation behaviour of surfactant depends on different physicochemical parameters, viz., temperature, $\mathrm{pH}$, additives, salinity and ionic strength ${ }^{1-4)}$. An ionic liquid (ILs) usually contains one cationic organic component and also consists of short lived ion pair, that facilitates interfacial adsorption and aggregation of conventional surfactants ${ }^{5-7)}$. Ionic liquids not only have emerged as alternatives to the conventional solvents, but it can also significantly alter surfactant aggregation ${ }^{8-10)}$. In case of ILs having melting point below $100^{\circ} \mathrm{C}$, mutual interfacial adsorption and aggregation take place along with the surfactants ${ }^{7}$. ILs can easily interact with surfactant molecules leading to the formation of mixed micelles ${ }^{11}$. Electrostatic interaction between two oppositely charged head groups can demonstrate different hydration energy for the surfactant molecules in presence of ionic liquid, especially for anionic surfactants ${ }^{12,13)}$. Oppositely charged IL

\begin{abstract}
+ surfactant mixtures exhibit strong synergistic interaction which are capable to exhibit superior interfacial properties, viz., higher surface activity, lower $C M C$, formation of a variety of aggregates ${ }^{5-7}$. Charge on such micellar aggregates, induced by IL are mainly governed by two parameters: i) extent of charge neutralization and ii) presence of counter ions ${ }^{14,15)}$. In general, IL + surfactant mixtures are capable to form various complex phases ${ }^{16)}$, like coacervates $^{17)}$, micelles ${ }^{18)}$, aggregates ${ }^{19)}$, and vesicles ${ }^{20)}$ that arise at different ILs concentration, as well as other environmental factors.

Having the ability of self organisation as micelle, certain ILs carrying long hydrocarbon chain possessing the amphiphilicity with distinct hydrophobic tail and hydrophilic head groups are called surface-active ionic liquids (SAILs) ${ }^{21)}$. In SAIL, either the surface active part or the counter ion can have an ionic liquid like component. SAILs possess the dual benefits of surfactant as well as ionic liquids.

The main aim of the present work is to understand the
\end{abstract}

*Correspondence to: Amiya Kumar Panda, Department of Chemistry, Vidyasagar University, Midnapore-721102, West Bengal, INDIA

E-mail: akpanda@mail.vidyasagar.ac.in

Accepted November 16, 2020 (received for review October 27, 2020)

Journal of Oleo Science ISSN 1345-8957 print / ISSN 1347-3352 online

http://www.jstage.jst.go.jp/browse/jos/ http://mc.manusriptcentral.com/jjocs 


\section{K. Mandal, M. Barai, H. Sultana et al.}

physicochemistry of SAILs. Towards the endeavour, initially it is considered worthy to replace the common cations in anionic surfactants with a cationic moiety of ionic liquids (e.g., 1-alkyl-3-methylimidazolium, 1-alkylpyridinium ion) . Aggregation behavior of conventional surfactants in IL + Water mixture need to be investigated in order to understand the aggregation behaviour of the aforementioned systems. For this reason, aggregation behavior of SDS was studied in aqueous solutions of two different ionic liquids, 1-butyl-3-methylimidazolium chloride $([\mathrm{bmim}] \mathrm{Cl})$ and $N$ butyl- $N$-methyl pyrrolidinium tetrafluoroborate ([bmp] $\left.\mathrm{BF}_{4}\right)$. These two ILs find applications in different fields that include enzymatic hydrolysis ${ }^{22)}$, adsorbents for gas separation ${ }^{23)}$, energy storage ${ }^{24)}$ and high power electric storage $^{25)}$, to mention a few.

Physicochemical investigation on the surface adsorption and aggregation behaviour of anionic surfactant sodium dodecyl sulphate (SDS) in aqueous-IL $(\mathrm{IL}+\mathrm{W})$ mixed solvent systems were carried out in presence of two ILs, 1-butyl-3-methylimidazolium chloride[bmim $] \mathrm{Cl}$, and $N$-butyl- $N$-methyl pyrrolidinium tetrafluoroborate $[\mathrm{bmp}] \mathrm{BF}_{4}$. The choices of the two ILs are specific as the two ILs mainly favour solvating properties differently due to their differences in the charge densities in their cationic moieties $^{5)}$. Both SDS based SAILs and SDS in water form micelles which were reported to be useful in drug delivery. Micelles of surfactant having lower CMC value, in the presence of IL, than that of only SDS in water are more suitable for practical applications ${ }^{12,13)}$. As the CMC values of SDS based SAILs and IL + surfactant mixtures were found to be comparable. IL + surfactant mixed micelles are thermodynamically and kinetically as stable as the micelles of SDS based SAILs. Moreover, simply mixing aqueous SDS with IL we get the desired mixed micelles. SDS finds to be suitable in a variety of applications, viz., fabrication of nanomateri$\mathrm{als}^{26)}$, tooth paste ${ }^{27)}$, detergents ${ }^{28)}$, and microemulsion formulation $^{29)}$, to mention a few. On the other hand, ILs find applications as lubricant ${ }^{30)}$, cleaning $^{31)}$, in food industries ${ }^{32)}$, microemulsion ${ }^{33)}$ and preparation of pharmaceutical products, etc. ${ }^{34)}$. Hence, IL + SDS mixed systems are considered to exhibit a wide range of applications, viz., biochemical research $^{35)}$, textile wetting ${ }^{32)}$, synthesis of nanoparticle ${ }^{26)}$, drug delivery ${ }^{36)}$, molecular separation ${ }^{37,38)}$, and cosmetics $^{39}$. Micelles can incorporate the bulky organic ions of ionic liquids by inciting the hydrophobic part of surfactant through electrostatic and hydrophobic interactions $\mathrm{s}^{40,41}$. ILs in water do not show micellization properties, staying in ionised condition like electrolytes therefore, starting from minimum range of ILs concentrations used in mixed micelles and looking for stable, leak proof micelles with lower CMC. Higher hydrophilicity of imidazolium or pyrrolidinium groups with SDS are quite different than that of the aggregation of mixed micelles, so using such surfactant with multitude of such ionic liquids are considered to be very important ${ }^{42}$. Studies were carried out by surface tension, electrical conductance, UV-VIS absorption and emission spectroscopy, and dynamic light scattering(DLS) studies $^{6,40,43)}$. Critical micelle concentration $(C M C)$, surface pressure at $C M C\left(\pi_{\mathrm{CMC}}\right)$ and Gibbs free energy change for micellization $\left(\Delta G_{\text {mic }}^{0}\right)$ values were obtained from surface tension data ${ }^{7}$. Degree of dissociation $(\alpha)$ values of the micellar aggregates were determined by analyzing the conductance data. Aggregation number of the micellar aggregates $(n)$ in presence of varying concentration of ILs were determined by fluorescence quenching method using pyrene(Py) as the probe and hexadecylpyridinium chloride (HPC) as the quencher. Hydrodynamic diameter $\left(d_{\mathrm{h}}\right)$, polydispersity index $(P D I)$ values were evaluated from the dynamic light scattering studies. Formation of aggregates leading the much more favourable usefulness towards human being as contributing different types of master's roles in medicinal purpose industrial purpose as well as in daily uses also ${ }^{5,10,40)}$. The aggregation behaviour and thermodynamics of the micellization processes have been studied to develop suitable drug delivery systems having high drug loading properties ${ }^{8}$, and templates/media for the synthesis of nanoparticles. Micellization behavior of IL + SDS mixtures exhibits new insight, would be eventually help to study the aggregation $^{44}$. Present research group is in a process to study the aggregation behaviour of counter ion free ionic liquid based surfactant. Prior to that, in order to understand the effect of the IL on the aggregation behaviour, such a set of studies have been undertaken.

\section{Experimental section}

\subsection{Materials}

Sodium dodecyl sulphate (SDS), 1-butyl-3-methylimidazolium chloride [bmim] Cl, $N$-butyl- $N$-methyl pyrrolidinium tetrafluoroborate $[\mathrm{bmp}] \mathrm{BF}_{4}$ were the products from SigmaAldrich Chemicals Pvt. Ltd. (St. Louis, MO, USA). AR grade pyrene, hexadecylpyridinium chloride (HPC), ethanol were purchased from Acros Organics Pvt. Ltd (Mumbai, India). 99\% pure chemicals were used as received; the chemicals were purified using the standard procedure. Double distilled water with specific conductance $2-4 \mu \mathrm{S} \mathrm{cm}^{-1}$ at 298 $\mathrm{K}$ was used in preparing the different experimental solutions. Structure of surfactant and ionic liquids are shown in Scheme 1.

\subsection{Methods}

2.2.1 Determination of critical micelle concentration (CMC)

$C M C$ values of SDS in water and IL $+\mathrm{W}$ mixtures were determined by surface tension, conductance, UV-VIS absorption/emission spectroscopy studies ${ }^{5-7,10)}$. 
<smiles>CCCOS(=O)(=O)[O-]</smiles>

Sodium dodecyl sulphate (SDS)

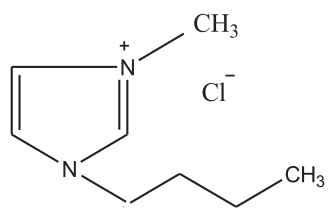

1-Butyl-3 methylimidazolium chloride $([\mathrm{bmim}] \mathrm{Cl})$

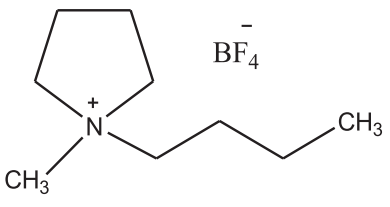

1-Butyl-3-methylpyrrolidinium tetrafluoroborate $\left([\mathrm{bmp}] \mathrm{BF}_{4}\right)$

Scheme 1 Chemical structure of SDS, $[\mathrm{bmim}] \mathrm{Cl}$ and $[\mathrm{bmp}] \mathrm{BF}_{4}$.

\subsubsection{Surface tension measurements}

Surfactant stock solutions (twenty times stronger than the anticipated CMC values) was prepared in pure water or corresponding ionic liquid + water $(\mathrm{IL}+\mathrm{W})$ mixed solvent systems at different ionic liquid concentration in water in the range of 0.025-2.5 mM. $20 \mathrm{~mL}$ of double distilled water or $(\mathrm{IL}+\mathrm{W})$ was taken in a container; temperature was controlled by circulation water bath (Hahntech Corporation, South Korea). Quantitative amount of surfactant mixture, dissolved in water or in water + IL mixed solvent systems, were progressively added using a Hamilton(USA) microsyringe to the respective solvent and were homogenized using a magnetic stirrer. Surface tension data was recorded by du Noüy tensiometer with a stated error of $\pm 0.1 \mathrm{mNm}^{-1}$ (Jencon, Kolkata, India) ${ }^{45-47)}$. CMC value was determined from the breakpoint of surface tension $(\gamma)$ vs. $\log [\mathrm{S}]$ plots ([S] being the surfactant concentration).

\subsubsection{Conductance studies}

Conductance studies were also carried out using the same protocol as adopted in the surface tension measurement. Specific conductance $(\kappa)$ of solution was measured by a direct reading conductivity meter, Cyber Scan CON510 (Eutech Instruments, Singapore) with an accuracy of $\pm 0.1 \mu \mathrm{Scm}^{-1}$. Conductance ( $\kappa$ ) vs. [S]plots, at a particular break point indicates $\mathrm{CMC}^{48)}$.

\subsubsection{UV-VIS absorption/emission spectroscopic studies}

Initially, $1 \mathrm{mM}$ pyrene solution was prepared in dehydrated ethanol. A $10^{-4} \mathrm{M}$ Py solution in ethanol was prepared by dilution; afterwards $2.5 \mu \mathrm{M}$ Py in water was prepared by proper dilution and sonication technique. UV-VIS spectrophotometer(UVD-2950, Labomed Inc., USA) instrument was used in recording the absorption spectra ${ }^{49)}$. Py displays four major absorption peaks at 241, 274, 337 and $345 \mathrm{~nm}$ and two weak bands at 263 and $321 \mathrm{~nm}$ (panel A, Fig. S1, supplementary section $)^{50)}$. The sum of the absorbance $\left(A_{\mathrm{T}}\right)$ vs. surfactant concentration $[\mathrm{S}]$ followed sigmoidal profiles; $C M C$ values were determined form the plot using the following expression ${ }^{46,50)}$ :

$$
A_{\mathrm{T}}=\frac{\left(a_{i}-a_{f}\right)}{\left[1+\exp \frac{\left(x-x_{0}\right)}{\Delta x}\right]}+a_{f}
$$

where, $x_{0}$ is centre of the sigmoid (herein the CMC), $x$ is the surfactant concentration, $a_{i}$ and $\alpha_{f}$ is initial and final asymptotes of the sigmoidal curve respectively, and $\Delta x$ is the interval of the independent variable. Fluorescence spectroscopic studies were also performed (Hitachi High Technologies Fluorescence Spectrophotometer Corporation, F-7100, Tokyo, Japan) to determine the CMC value of SDS under varied concentration of ILs. First vibronic emission peak at $375 \mathrm{~nm}\left(I_{1}\right)$ and the third vibronic emission peak at $385 \mathrm{~nm}\left(I_{3}\right)$ of Py were considered for emission studies. (panel B, Fig. S1) ${ }^{50)}$. Py was excited at $337 \mathrm{~nm}$ and the emission spectra were recorded in the range 350 to 450 nm. From the midpoint of the sigmoidal profile of the $I_{1} / I_{3}$ vs. $[\mathrm{S}]$ plot, $C M C$ values were determined ${ }^{46)}$. 2.2.5 Dynamic light scattering(DLS) studies

A Zetasizer Nano ZS-90 (Malvern Instruments, U.K.) dynamic light scattering spectrometer was used to determine the size and polydispersity index $(P D I)$ of micellar aggregates $^{51)}$. He-Ne laser operating light $(632.8 \mathrm{~nm})$ was used for DLS measurements and the data were collected at a scattering angle of $90^{\circ}$. Ten times concentrated surfactant solutions (than its CMC value) were used for DLS studies. Surfactant mixtures were filtered through $0.45 \mu \mathrm{m}$ Millipore cellulose acetate membrane filter prior to recording the data. Viscosity of the medium was measured by a DV II-Pro roto viscometer (Brookfield, USA) with a stated accuracy of $\pm 0.01 \mathrm{cP}^{52)}$. Viscosity coefficient was measured at different shear rate and extra plotted the zero shear viscosity was evaluated from the plot of viscosity vs. shear rate. Refractive index of the medium was measured by an Abbe type refractometer ${ }^{53)}$. Experimental value of the viscosity coefficient and refractive index of the solution under investigation, as measured above were taken into account while performing the DLS studies.

All experiments were carried out at $298 \mathrm{~K}$.

\section{Results and Discussion}

\subsection{Critical Micelle Concentration ( $C M C$ )}

$C M C$ value of SDS at different concentration of ILs in water were evaluated by analyzing the surface tension, conductance, UV-visible absorption and emission spectroscopic data at different concentration of SDS. Representative profiles for determining the $C M C$ values of SDS are shown in Fig. $1^{5,6,40)}$. $C M C$ values (reported herein as the average $C M C$ determined by all the aforementioned 


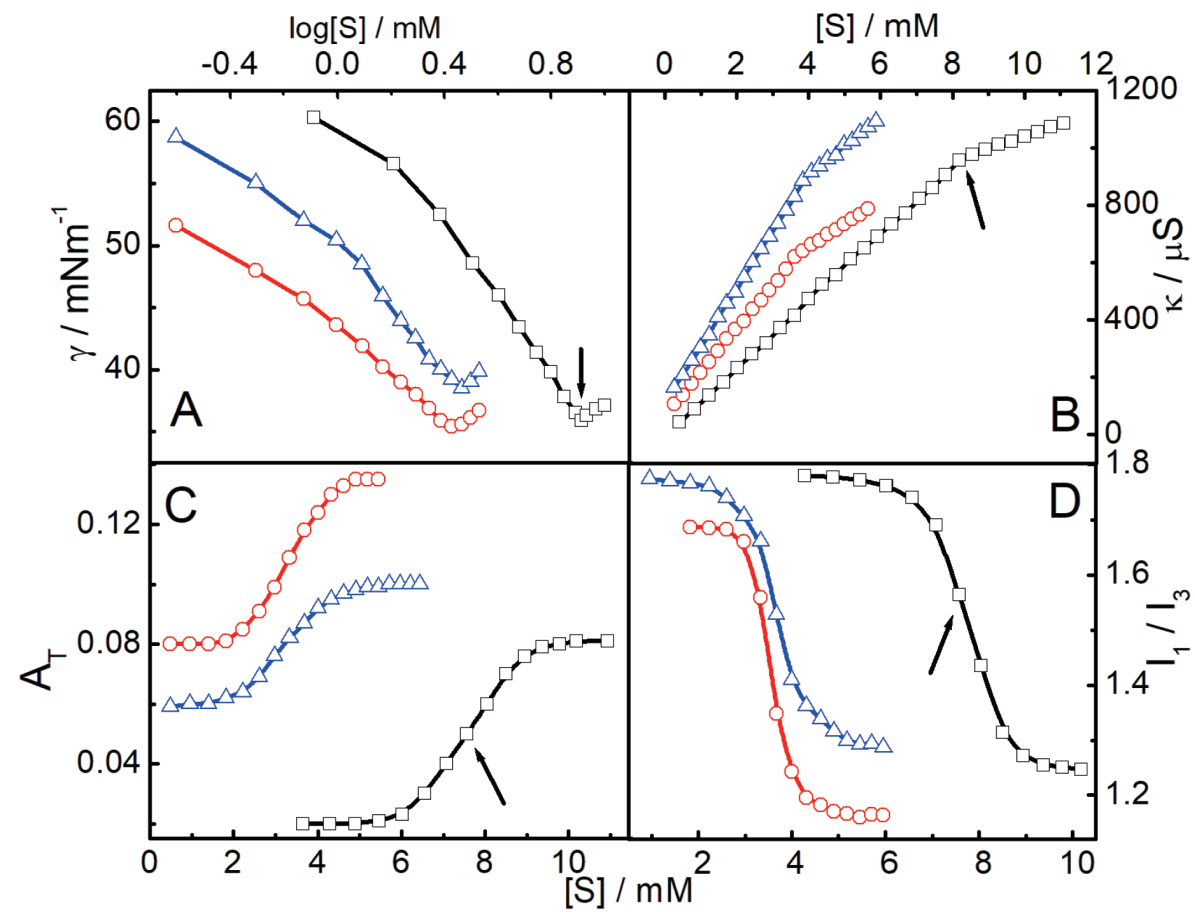

Fig. 1 Variation of surface tension $(\gamma, \mathrm{A})$, specific conductance $(\kappa, \mathrm{B})$, sum of the peak absorbance $\left(\mathrm{A}_{\mathrm{T}}, \mathrm{C}\right)$, and $I_{1} / I_{3}$ ratio $(\mathrm{D})$ with SDS concentration ( $[\mathrm{S}]$ ) at $298 \mathrm{~K}$. $[\mathrm{Py}]=2.5 \mu \mathrm{M}$. $\lambda_{\mathrm{ex}}=337 \mathrm{~nm}$. Systems: $\square$, water; $\bigcirc,[\mathrm{bmim}] \mathrm{Cl}+\mathrm{W}$ and $\Delta$, $[\mathrm{bmp}] \mathrm{BF}_{4}+\mathrm{W}$. Concentration of ionic liquid in water, [IL] $=0.25 \mathrm{mM}$.

Table 1 Variation of $C M C$, surface pressure at $C M C\left(\pi_{\mathrm{CMC}}\right)$, Gibbs free energy of micellization $\left(\Delta G_{\text {mic }}^{0}\right)$, fraction of counter ion binding $(\beta)$ and aggregation number $(\mathrm{n})$ of SDS with the variation of ionic liquid concentration in water at $298 \mathrm{~K}$.

\begin{tabular}{|c|c|c|c|c|c|c|c|c|c|}
\hline \multirow[b]{2}{*}[\mathrm{IL}]{$/ \mathrm{mM}$} & \multicolumn{5}{|c|}{$C M C / \mathrm{mM}$} & \multirow[b]{2}{*}{$\begin{array}{c}\pi_{\mathrm{CMC}} \\
\mathrm{mNm}^{-1}\end{array}$} & \multirow{2}{*}{$\begin{array}{c}(-) \\
\Delta G_{\mathrm{mic}}^{0} / \mathrm{kJ} \\
\mathrm{mol}^{-1}\end{array}$} & \multirow{2}{*}{$\begin{array}{c}\beta= \\
\left(1-S_{2} /\right. \\
\left.S_{1}\right)\end{array}$} & \multirow[b]{2}{*}{$n$} \\
\hline & $\begin{array}{l}\text { Surface } \\
\text { tension }\end{array}$ & Conductance & $\begin{array}{l}\text { UV-vis } \\
\text { abs. }\end{array}$ & Fluorescence & Average & & & & \\
\hline 0 (water) & 8.0 & 8.1 & 7.6 & 7.8 & 7.7 & 37.0 & 17.7 & 0.42 & 73 \\
\hline \multicolumn{10}{|c|}{$[\mathrm{bmim}] \mathrm{Cl}$} \\
\hline 0.025 & 3.6 & 4.0 & 3.9 & 4.0 & 3.72 & 36.4 & 18.5 & 0.40 & 90 \\
\hline 0.225 & 3.1 & 3.9 & 3.5 & 3.7 & 3.38 & 36.8 & 19.4 & 0.41 & 130 \\
\hline 0.250 & 2.7 & 3.5 & 3.2 & 3.5 & 3.02 & 37.7 & 21.0 & 0.57 & 133 \\
\hline 1.375 & 2.5 & 2.7 & 2.5 & 2.4 & 2.44 & 39.0 & 22.9 & 0.43 & 134 \\
\hline 2.250 & 2.2 & 2.6 & 2.4 & 2.1 & 2.24 & 38.8 & 23.6 & 0.60 & 148 \\
\hline 2.500 & 2.0 & 2.4 & 2.1 & 2.0 & 2.06 & 39.2 & 24.4 & 0.63 & 161 \\
\hline \multicolumn{10}{|c|}{$[\mathrm{bmp}] \mathrm{BF}_{4}$} \\
\hline 0.025 & 3.8 & 4.3 & 4.1 & 4.1 & 3.90 & 34.7 & 18.1 & 0.37 & 78 \\
\hline 0.225 & 3.4 & 3.9 & 3.5 & 3.9 & 3.48 & 34.4 & 19.0 & 0.38 & 80 \\
\hline 0.250 & 3.0 & 3.7 & 3.3 & 3.6 & 3.18 & 34.6 & 20.2 & 0.42 & 102 \\
\hline 1.375 & 2.6 & 3.1 & 3.1 & 2.7 & 2.74 & 36.3 & 21.3 & 0.49 & 134 \\
\hline 2.250 & 2.4 & 2.9 & 2.7 & 2.3 & 2.46 & 36.4 & 22.7 & 0.57 & 166 \\
\hline 2.500 & 2.2 & 2.7 & 2.3 & 2.2 & 2.26 & 37.6 & 23.5 & 0.60 & 170 \\
\hline
\end{tabular}

methods) of SDS gradually decreases with increasing ILs concentration in water (Table 1).

$C M C$ of SDS in water as well as in $\mathrm{IL}+\mathrm{W}$ is one of the important parameters primarily in understanding the aggregation behaviour of mixed micelles. $C M C$ value of SDS in water is $7.7 \mathrm{mM}$ close to literature value ${ }^{45}$. $C M C$ value of 


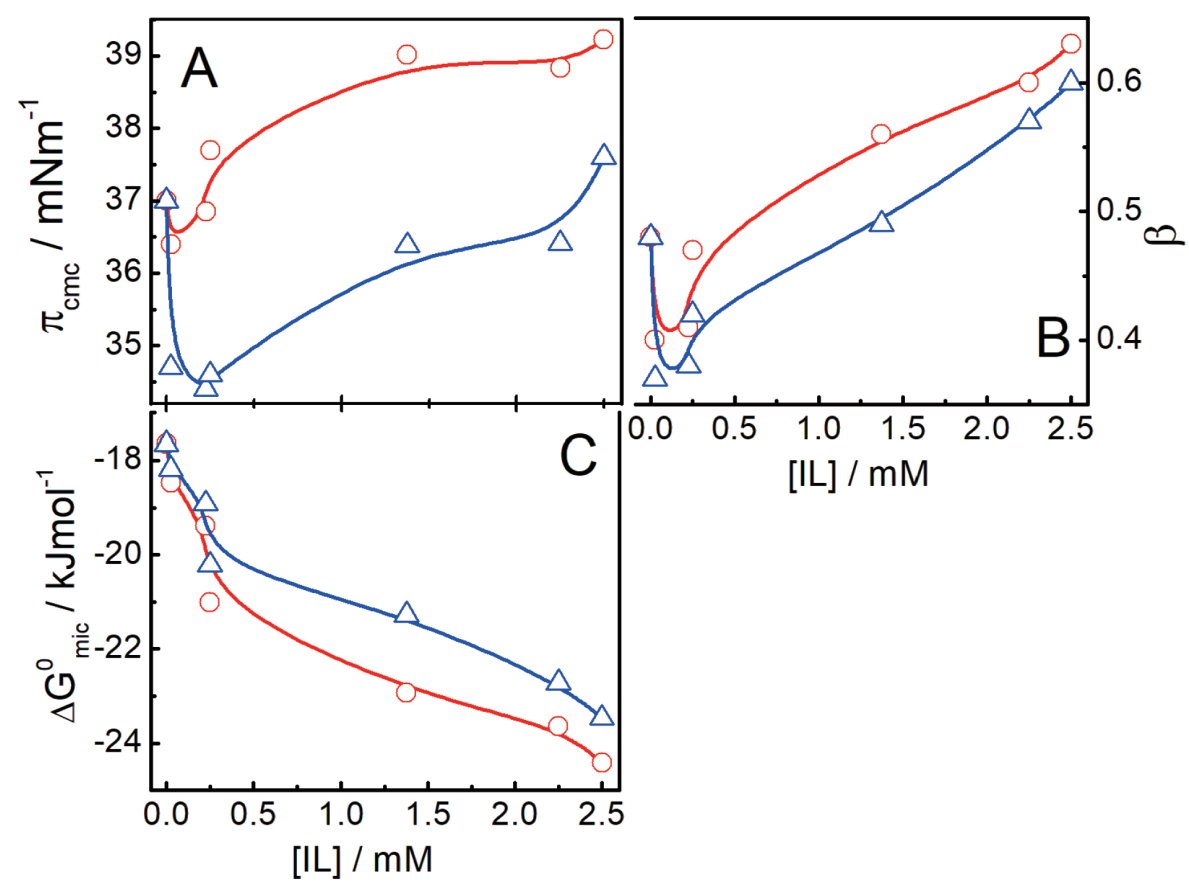

Fig. 2 Variation of surface pressure at the $C M C\left(\pi_{\mathrm{cmc}}, \mathrm{A}\right)$, fraction of counter ion binding $(\beta, \mathrm{B})$ and Gibbs free energy of micellization $\left(\Delta G_{\text {mic }}^{0}\right.$, C) with the concentration of ionic liquid[IL] in water at $298 \mathrm{~K}$. Systems: $\bigcirc,[\mathrm{bmim}] \mathrm{Cl}$ and $\Delta$, $[\mathrm{bmp}] \mathrm{BF}_{4}$.

SDS in $2.5 \mathrm{mM}[\mathrm{bmim}] \mathrm{Cl}$ and $[\mathrm{bmp}] \mathrm{BF}_{4}$ were $2.06 \mathrm{mM}$ and $2.26 \mathrm{mM}$ respectively. The effect of $[\mathrm{bmim}]^{+}$on the $\mathrm{CMC}$ value of SDS is more profound than $[\mathrm{bmp}]^{+}$due to higher the polarizability/charge density on the imidazolium ring than the pyrrolidinium moiety, that stabilizes/neutralizes the charge of the anionic surfactant promoting the micellization at lower concentration. Besides, the presences of a butyl side chain in both the ionic liquids are expected to form some sort of mixed micelles ${ }^{12,13)}$.

\subsection{Interfacial behaviour}

Interfacial behaviour of SDS in IL $+\mathrm{W}$ mixed solvent systems were investigated, which were then compared with that of SDS in water.

Surface pressure at $C M C\left(\pi_{\mathrm{cmc}}\right)$ values were determined for both the imidazolium and pyrrolidinium based IL $+\mathrm{W}$ mixed solvent systems at different concentrations by using the following equation ${ }^{5,6,40,45,55)}$ :

$$
\pi_{\mathrm{cmc}}=\gamma_{\mathrm{S}}-\gamma_{\mathrm{cmc}}
$$

where, the $\gamma_{\mathrm{s}}$ and $\gamma_{\mathrm{cmc}}$ are the surface tension of pure solvent (either pure water or IL + water mixture) and the same for a surfactant solution at the $C M C$ value of surfactant solution. For both the ionic liquids with increasing concentration, $\pi_{\mathrm{cmc}}$ values progressively passed through minima which was graphically shown in Fig. 2(panel A). Results suggest that the ILs initially increases the hydrophobicity of the medium, thus assisting adsolubilization of surfactant, beyond a certain concentration, the ILs tender similar effects as that of the normal salts. The $\pi_{\mathrm{cmc}}$ values vary in the range $36.4-39.2 \mathrm{mN} \mathrm{m}^{-1}$ and $34.7-37.6 \mathrm{mN} \mathrm{m}^{-1}$ for imidazolium and pyrrolidinium based IL respectively (Table 1). Due to higher polarizability of imidazolium ring the effect was more pronounced than the pyrrolidinium based IL.

\subsection{Conductance studies}

Conductance measurements were carried out to determine the $C M C$ values and the fraction of counter-ion dissociation $(\alpha)$ of the SDS in IL + W mixed solvent systems. The slope of post $\left(S_{2}\right)$ - and pre $\left(S_{1}\right)$-micellar regions can effectively be used in evaluating the fraction of counter ion dissociation $(\alpha)$ values vide the following equation ${ }^{45,56}$ :

$$
\alpha=\frac{S_{2}}{S_{1}}
$$

The $\alpha$ values of SDS in both the IL $+\mathrm{W}$ mixed systems initially increases and passes through maxima which then decrease monotonously with increasing IL concentration, as shown in Fig. 2 (panel B). The $\alpha$ value of SDS only in water is found to be 0.58 , that was comparable with the literature values ${ }^{45,54}$. For both the ILs, the $\alpha$ values pass through maxima further supporting the proposition that initially the ILs help in the adsolubilization of SDS, beyond a certain concentration the effects of ILs become similar to the conventional salts in water; the only major difference lies in the fact that unlike the conventional salts, IL cations can form mixed micelles with the SDS where the IL cations also act as counter ions ${ }^{12,13)}$. While describing the fraction 
of counter ion binding, evaluated by analyzing the conductance data, it is proposed that a substantial fraction of ionic liquid cations can act as the counter ions to the anionic micelles. The fraction of counter ion binding ( $\beta$, as presented in Table 1) increased from 0.4 in water to $\sim 0.6$ in presence of $2.5 \mathrm{mM}$ aqueous ILs in water. It is proposed that the increased $\beta$ value is due to the incorporation of ionic liquid cations onto the anionic micelles as the counter ion. This proposition was further supported by the DLS data, as discussed later. However further investigations involving the structural parameters are warranted in order to confirm the proposition.

\subsection{Thermodynamics of micellization}

Gibbs free energy of micellization $\left(\Delta G_{\text {mic }}^{0}\right)$ values were evaluated using the following expressions ${ }^{46,57-61)}$ :

$$
\Delta G_{\text {mic }}^{0}=(2-\alpha) \text { RT } \ln X_{\text {cmc }}
$$

where, $X_{\mathrm{CMC}}$ is the mole fraction of surfactant (the other component being the solvent) at the $C M C, \mathrm{R}$ and $\mathrm{T}$ have their usual significances. Negative values of $\Delta G_{\text {mic }}^{0}$ indicate spontaneity of micellization processes, shown in Fig. 2 (panel C). The $\Delta G_{\text {mic }}^{0}$ value for SDS in water is found to be $-17.7 \mathrm{~kJ} \mathrm{~mol}^{-1}$ similar to the earlier reported values ${ }^{45,54)}$. With increasing IL concentration in water the magnitude of the $\Delta G^{0}$ mic values increase is more prominent in case of [bmim] $\mathrm{Cl}\left(-18.5\right.$ to $\left.-24.4 \mathrm{~kJ} \mathrm{~mol}^{-1}\right)$ than the $[\mathrm{bmp}] \mathrm{BF}_{4}+$ W system $\left(-18.1\right.$ to $\left.-23.5 \mathrm{~kJ} \mathrm{~mol}^{-1}\right)$ further supporting the earlier proposition. From the $\beta$ values of SDS in mixed solvent systems, it can be concluded that the extent of dissociation of surfactant molecules occur more spontaneously with increasing polarity of the medium.

\subsection{Micellar aggregation number}

Aggregation number of SDS (n) in water and as well as in $\mathrm{IL}+\mathrm{W}$ mixed solvent systems were determined using Py as probe and HPC as quencher $(\mathrm{Q})$ using the following equa$\operatorname{tion}^{43,46,60,61)}$ :

$$
\ln \frac{I_{0}}{I}=\mathrm{n} \frac{[\mathrm{Q}]}{[\mathrm{S}]-\mathrm{CMC}}
$$

where, $I_{0}$ and $I$ are the fluorescence intensity of pyrene in the absence and presence of $\mathrm{Q}$ respectively, in the presence of a particular surfactant concentration[S]. The third vibronic emission peak of Py $(385 \mathrm{~nm})$ was taken into account to determine the aggregation number (n) of SDS. Some representative $\ln I_{0} / I$ vs. quencher concentration [Q] plots have been shown in Fig. S2 (supplementary section). Aggregation number of SDS in pure water is 73, similar to the literature value ${ }^{45}$. Micellar aggregation number of SDS gradually increases with increasing concentration of IL; in case of $[\mathrm{bmim}] \mathrm{Cl}$ the values increase in the range of 90 to 161 while for [bmp] $\mathrm{BF}_{4}$ it ranged in between 78 to 170 (Fig. 3, panel A (also summarized in Table 1, along with other results)). Increased aggregation number of SDS in IL + W

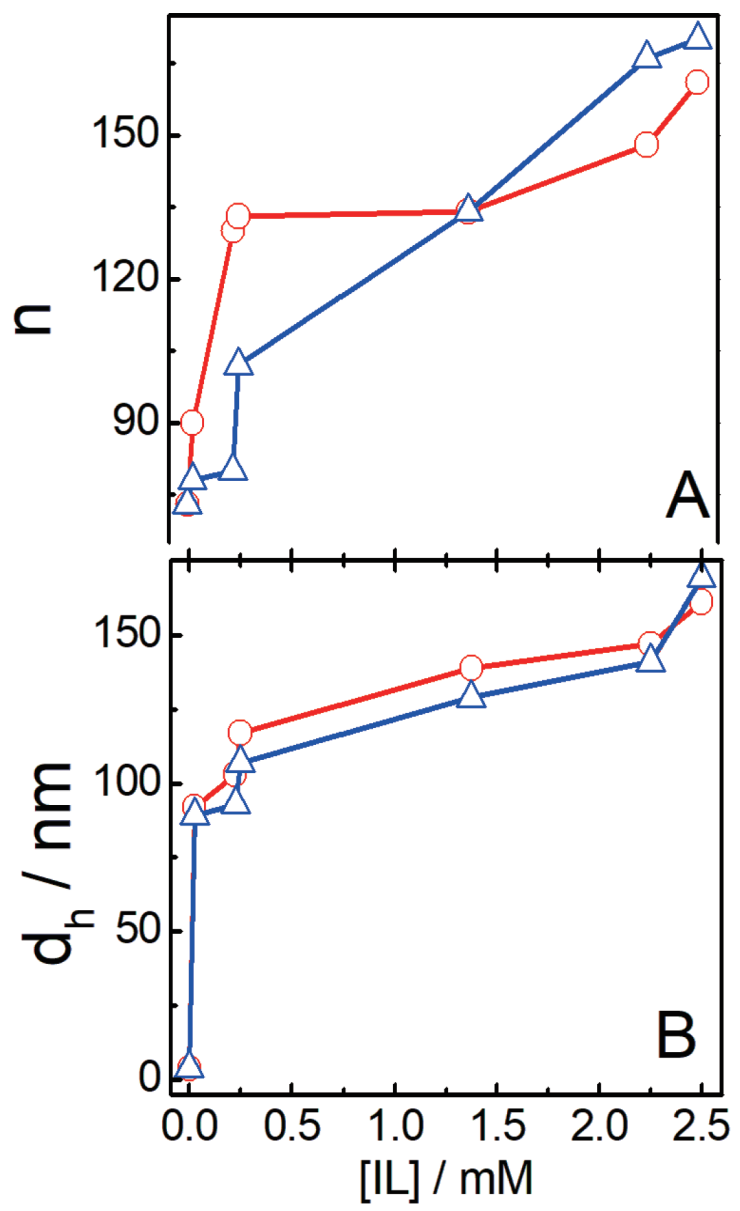

Fig. 3 Variation of aggregation number ( $n, A)$ and hydrodynamic diameter $\left(d_{h}, B\right)$, of SDS micelles with the concentration of ionic liquid[IL] in water at $298 \mathrm{~K}$. Systems: $\bigcirc,[\mathrm{bmim}] \mathrm{Cl}$ and $\Delta,[\mathrm{bmp}] \mathrm{BF}_{4}$.

mixed solvent systems compared to pure water are due to the presence of ionic liquid molecules in water which increases the polarity of the mixed systems; also it is known that the micellar aggregation number increases with increasing salt concentration ${ }^{43,46,60,61)}$. Almost two fold increases in micellar aggregation number in presence of ionic liquids are due to the formation of mixed micelles between the dodecyl sulphate anion and ionic liquid cations that interact favourably through electrostatic attraction. Besides, the butyl moieties of the ionic liquid cations can easily get anchored on to the palisade layer of the anionic micelles, as also evidenced by a 1.5 times increase in the fraction of counter ion binding in presence of ILs, obtained from the conductance data. The proposition was further supported by the DLS data as discussed below.

\subsection{Dynamic light scattering(DLS) studies}

Hydrodynamic diameter $\left(d_{\mathrm{h}}\right)$ and polydispersity index $(P D I)$ values of SDS micelles in different IL $+\mathrm{W}$ media were measured by DLS studies. Size of SDS in pure water is 
found to be $3.8 \mathrm{~nm}$, close to literature value ${ }^{45,55)}$. Size of SDS micelles increased sigmoidally with increasing IL concentration in water (Fig. 3, along with other data). $d_{\mathrm{h}}$ varied in the range $92-161 \mathrm{~nm}$ for $[\mathrm{bmim}] \mathrm{Cl}+\mathrm{W}$ while for [bmp $\mathrm{BF}_{4}$ it varied in the range 89-169 nm. Increase in micellar size with increasing IL concentration is driven by the strong electrostatic interaction between the IL cation and the anionic surfactant; the charge neutralization eventually increases the hydrophobicity of the medium that effectively increases the micellar size ${ }^{51)}$. Besides, after a threshold concentration, the effect of IL becomes similar to the conventional electrolytes, as already mentioned earlier. The charge density in imidazolium is higher than that of pyrrolidinium ring; hence it imparts higher polarity to SDS. The hydrophobic interaction into the micelles as well as dominant of SDS in micelles is the main factor for formation of larger aggregates. From the surface tension measurements it was observed that with increasing IL concentration the molecular cross sectional area increases. Hence it is quite expected that the micellar size of SDS (partly the ionic liquid molecules also take part in the micellization process). Thus the observations from the surface tension data, aggregation number and dynamic light scattering data could be correlated in explaining the variation of different physicochemical parameters of SDS aggregates in varying concentration of IL in water. Size and aggregation number of mixed micelles at the certain concentration of ILs were maximum, i.e., aggregate tendency higher has been shown up to certain concentration of ILs (almost about at $1 \mathrm{mM}$ conc.). It is believed that the increases in micellar size in presence of ILs are due to the incorporation/coadsorption of ionic liquid cations to the anionic micellar surface.

Polydispersity index $(P D I)$ value of SDS in water was found to be greater than 0.4 although count rate of photon was comparatively low. In presence of ILs, heterogeneous aggregates were formed, as reflected through the nonsystematic variation of $P D I$ values with the variation of ILs concentration (data not shown). As together the equilibrium area per surfactant molecule and surfactant tail length were higher in value than that of surfactant tail volume at aggregate interface occurring the resultant mixed micelles were spherical in shape, the results could be well correlated with aggregation number. However, further studies like, rheology measurements, small angle X-ray scattering, small angle neutron scattering, cryo-electron microscopy are warranted to envisage the structure of the aggregates.

\section{Conclusion}

In this work a bunch of experiments have been carried out to understand the interfacial and micellar aggregation behaviour of SDS in water as well as in $[\mathrm{bmim}] \mathrm{Cl}+\mathrm{W}$ and [bmp $] \mathrm{BF}_{4}+\mathrm{W}$ mixed solvent systems at different concentration of IL. Also the comparative studies have been done between imidazolium and pyrrolidinium based ionic liquid in water as mixed solvent systems. The lower $C M C$ values of those mixed micelles indicate hydrophobic interaction between SDS and cationic head groups of ILs. $C M C$ values of SDS in presence of imidazolium based IL are lower than pyrrolidinium based IL that is contributed by the high polarity of ionic liquid head group ${ }^{12,13)}$. The change in conductance value in different mixed micelle systems, leading to the negative values of $\Delta G^{0}$ mic indicate spontaneity of the aggregation process. Combined hydrogen bonding and electrostatic interaction between SDS and ILs in water, $\pi_{\mathrm{cmc}}$ of mixed micelle systems pass through minima which lead to the associative interaction at the micellar surface. Dissociation of SDS molecules lead the high charge density at the micelle surface, as reflected from the fraction of counter ion dissociation values. Larger size of micelles are the results of the formation of larger aggregates induced by the cationic moieties of ILs that form some sort of mixed micelles with the anionic surfactant ${ }^{12,13)}$. Interfacial and aggregation behavior of SDS in aqueous-ionic liquid mixed solvent systems are considered to be important in electrostatic differentiation in molecular level of the particles as well as in nanoparticle synthesis; such systems can also be used as drug delivery systems. In order to explicitly understand the effect of two different ionic liquids, studying the micellization of a surfactant with two ionic liquids comprising similar counter anion is considered to be worthy. Besides the effect of ionic liquids' counter anions should also be investigated by studying the miceillization in presence of corresponding conventional salts; these are considered as the future perspectives.

\section{Conflict of Interest}

Authors declare that there is no conflict of interests.

\section{Acknowledgments}

This work financially supported by the research grant from CSIR, New Delhi. A.K.P. sincerely acknowledges financial support in the form of a research grant from the CSIR, New Delhi (Project Ref. Number: 01 (2864)/16/EMRII) . Financial supports from the University Grants Commission(UGC), New Delhi, India through a UGC-BSR scheme (F.25-1/2014-15 (BSR)/7-234/2009 (BSR)), UGC-SAP (No. F. 5-9/ 2015/DRS-II (SAP-II)) and Department of Science and Technology DST(DST-FIST grants, No. SR/FST/CSI235/2011 (G) and SR/FST/CS-I/2017/7 (G)). We thank Dr. Mithun Roy for his help in DLS measurement at NIT Manipur. 


\section{Supporting Information}

This material is available free of charge via the Internet at http://dx.doi.org/jos.70.10.5650/jos.ess20303

\section{References}

1) Edlund, H.; Sadaghiani, A.; Khan, A. Phase behavior and phase structure for catanionic surfactant mixtures: dodecyltrimethylammonium chloride-sodium nonanoate-water system. Langmuir 13, 4953-4963 (1997).

2) Kronberg, B. Surfactant mixtures. Cur. Opin. Colloid Interface Sci. 2, 456-463(1997).

3) Moulik, S.P.; Ghosh, S. Surface chemical and micellization behaviours of binary and ternary mixtures of amphiphiles (Triton X-100, Tween-80 and CTAB) in aqueous medium. J. Mol. Liq. 72, 145-161(1997).

4) Shiloach, A.; Blankschtein, D. Prediction of critical micelle concentrations and synergism of binary surfactant mixtures containing zwitterionic surfactants. Langmuir 13, 3968-3981 (1997).

5) Bharatiya, B.; Hassan, P.; Sastry, N. Formulation of pyridinium based RTIL-in-cyclohexane microemulsions: Investigations on size, conductivity and molecular interactions. J. Mol. Liq. 218, 586-594(2016).

6) Pal, A.; Pillania, A. Effect of ionic liquids on surface and aggregation properties of non-ionic surfactant Triton $^{\mathrm{TM}}$ X-100 in aqueous media. J. Surfact. Deterg. 19, 1189-1198(2016).

7) Pal, A.; Chaudhary, S. Ionic liquid induced alterations in the physicochemical properties of aqueous solutions of sodium dodecylsulfate(SDS). Colloids Surf. A 430, 58-64 (2013).

8) Hollamby, M.J.; Tabor, R.; Mutch, K.J.; Trickett, K.; Eastoe, J.; Heenan, R.K.; Grillo, I. Effect of solvent quality on aggregate structures of common surfactants. Langmuir 24, 12235-12240 (2008).

9) Joshi, T.; Bharatiya, B.; Kuperkar, K. Micellization and interaction properties of aqueous solutions of mixed cationic and nonionic surfactants. J. Disp. Sci. Technol. 29, 351-357 (2008).

10) Modaressi, A.; Sifaoui, H.; Mielcarz, M.; Domańska, U.; Rogalski, M. Influence of the molecular structure on the aggregation of imidazolium ionic liquids in aqueous solutions. Colloids Surf. A 302, 181-185 (2007).

11) Wasserscheid, P.; Keim, W. Ionic liquids-new "solutions" for transition metal catalysis. Angew. Chem. 39, 3772-3789 (2000).

12) Behera, K.; Pandey, S. Concentration-dependent dual behavior of hydrophilic ionic liquid in changing properties of aqueous sodium dodecyl sulfate. J. Phys. Chem. B 111, 13307-13315(2007).

13) Behera, K.; Pandey, S. Modulating properties of aque- ous sodium dodecyl sulfate by adding hydrophobic ionic liquid. J. Colloid Interface Sci. 316, 803-814 (2007).

14) Horbaschek, K.; Hoffmann, H.; Hao, J. Classic L- $\alpha$ phases as opposed to vesicle phases in cationic-anionic surfactant mixtures. J. Phys. Chem. B 104, 2781$2784(2000)$.

15) Marques, E.F. Size and stability of catanionic vesicles: Effects of formation path, sonication, and aging. Langmuir 16, 4798-4807 (2000).

16) Herrington, K.L.; Kaler, E.W.; Miller, D.D.; Zasadzinski, J.A.; Chiruvolu, S. Phase behavior of aqueous mixtures of dodecyltrimethylammonium bromide (DTAB) and sodium dodecyl sulfate(SDS). J. Phys. Chem. 97, 13792-13802(1993).

17) Barker, C.A.; Saul, D.; Tiddy, G.J.T.; Wheeler, B.A.; Willis, E. Phase structure, nuclear magnetic resonance and rheological properties of viscoelastic sodium dodecyl sulphate and trimethylammonium bromide mixtures. J. Chem. Soc. Farad. Trans. 1 70, 154-162 (1974).

18) Jokela, P.; Jönsson, B.; Wennerström, H. Phase equilibria in systems containing both an anionic and a cationic amphiphile. A thermodynamic model calculation. in Book Phase equilibria in systems containing both an anionic and a cationic amphiphile. A thermodynamic model calculation. Steinkopff, Darmstadt, Ch. pp. 17-22 (1985).

19) Vethamuthu, M.S.; Almgren, M.; Brown, W.; Mukhtar, E. Aggregate structure, gelling, and coacervation within the $\mathrm{L}_{1}$ phase of the quasi-ternary system alkyltrimethylammonium bromide-sodium desoxycholate-water. $J$. Colloid Interface Sci. 174, 461-479 (1995).

20) Kondo, Y.; Uchiyama, H.; Yoshino, N.; Nishiyama, K.; Abe, M. Spontaneous vesicle formation from aqueous solutions of didodecyldimethylammonium bromide and sodium dodecyl sulfate mixtures. Langmuir 11, 2380-2384 (1995).

21) Nandwani, S.K.; Chakraborty, M.; Gupta, S. Adsorption of surface active ionic liquids on different rock types under high salinity conditions. Sci. Rep. 9, 14760 (2019).

22) Liu, L.; Chen, H. Enzymatic hydrolysis of cellulose materials treated with ionic liquid[BMIM] Cl. Chin. Sci. Bull. 51, 2432-2436 (2006).

23) Sun, Y.; Schemann, A.; Held, C.; Lu, X.; Shen, G.; Ji, X. Modeling thermodynamic derivative properties and gas solubility of ionic liquids with ePC-SAFT. J. Ind. Eng. Chem. Res. 58, 8401-8417 (2019).

24) Neale, A.R.; Schütter, C.; Wilde, P.; Goodrich, P.; Hardacre, C.; Passerini, S.; Balducci, A.; Jacquemin, J. Physical-chemical characterization of binary mixtures of 1-butyl-1-methylpyrrolidinium bis ( (trifluoromethyl) sulfonyl)imide and aliphatic nitrile solvents as po- 
tential electrolytes for electrochemical energy storage applications. J. Chem. Eng. Data 62, 376-390 (2017).

25) Seddon, K.R. Ionic liquids for clean technology. J. Chem. Technol. Biotechnol. Int. Res. Process. Environ. Clean Technol. 68, 351-356(1997).

26) Yingju, F.; Zhipeng, L.; Le, W.; Jinhua, Z. Catanionicsurfactant-controlled morphosynthesis and gas-sensing properties of corundum-type $\mathrm{In}_{2} \mathrm{O}_{3}$. Nanotechnol. 20, 285501 (2009).

27) Goddard, E.D. Polymer/surfactant interaction-Its relevance to detergent systems. J. Am. Oil Chem. Soc. 71, 1-16 (1994).

28) Bera, A.; Ojha, K.; Mandal, A. Synergistic effect of mixed surfactant systems on foam behavior and surface tension. J. Surfact. Deterg. 16, 621-630(2013).

29) Hait, S.; Majhi, P.; Blume, A.; Moulik, S.P. A critical assessment of micellization of sodium dodecyl benzene sulfonate (SDBS) and its interaction with poly (vinyl pyrrolidone) and hydrophobically modified polymers. JR 400 and LM 200. J. Phys. Chem. B 107, 3650-3658 (2003).

30) Butt, H.-J. Measuring electrostatic, van der Waals, and hydration forces in electrolyte solutions with an atomic force microscope. Biophys. J. 60, 1438-1444 (1991).

31) Carpena, P.; Aguiar, J.; Bernaola-Galván, P.; Carnero Ruiz, C. Problems associated with the treatment of conductivity-concentration data in surfactant solutions: Simulations and experiments. Langmuir 18, 6054-6058(2002).

32) Lioi, S.B.; Wang, X.; Islam, M.R.; Danoff, E.J.; English, D.S. Catanionic surfactant vesicles for electrostatic molecular sequestration and separation. Phys. Chem. Chem. Phys. 11, 9315-9325(2009).

33) Ray, G.B.; Chakraborty, I.; Ghosh, S.; Moulik, S.; Palepu, R. Self-aggregation of alkyltrimethylammonium bromides $\left(\mathrm{C}_{10^{-}}, \mathrm{C}_{12^{-}}, \mathrm{C}_{14^{-}}\right.$, and $\left.\mathrm{C}_{16} \mathrm{TAB}\right)$ and their binary mixtures in aqueous medium: A critical and comprehensive assessment of interfacial behavior and bulk properties with reference to two types of micelle formation. Langmuir 21, 10958-10967 (2005).

34) Wilkinson, J.M.; Hipwell, M.; Ryan, T.; Cavanagh, H.M.A. Bioactivity of Backhousia citriodora: Antibacterial and antifungal activity. J. Agric. Food Chem. 51, 76-81 (2003).

35) Brackman, J.C.; Engberts, J.B.F.N. Polymer-micelle interactions: physical organic aspects. Chem. Soc. Rev. 22, 85-92(1993).

36) Boudier, A.; Castagnos, P.; Soussan, E.; Beaune, G.; Belkhelfa, H.; Ménager, C.; Cabuil, V.; Haddioui, L.; Roques, C.; Rico-Lattes, I.; Blanzat, M. Polyvalent catanionic vesicles: Exploring the drug delivery mechanisms. Int. J. Pharm. 403, 230-236 (2011).

37) Panda, S.; Kundu, K.; Singh, A.P.; Senapati, S.; Gardas,
R.L. Understanding differential interaction of protic and aprotic ionic liquids inside molecular confinement. J. Phys. Chem. B 121, 9676-9687(2017).

38) Trappe, V.; Sandkühler, P. Colloidal gels-low-density disordered solid-like states. Cur. Opin. Colloid Interface Sci. 8, 494-500 (2004).

39) Guaratini, T.; Gianeti, M.D.; Campos, P.M.B.G.M. Stability of cosmetic formulations containing esters of vitamins $\mathrm{E}$ and A: Chemical and physical aspects. Int. J. Pharm. 327, 12-16 (2006).

40) Anton, N.; Pierrat, P.; Lebeau, L.; Vandamme, T.F.; Bouriat, P. A study of insoluble monolayers by deposition at a bubble interface. Soft Matter 9, 10081-10091 (2013).

41) Rogers, R.D.; Seddon, K.R. Ionic liquids-solvents of the future? Science 302, 792-793(2003).

42) Plechkova, N.V.; Seddon, K.R. Applications of ionic liquids in the chemical industry. Chem. Soc. Rev. 37, 123-150 (2008).

43) Pal, A.; Pillania, A. Aggregation behavior of task-specific acidic ionic liquid $N$-methyl-2-pyrrolidinium dihydrogen phosphate $[\mathrm{nmp}]\left[\mathrm{H}_{2} \mathrm{PO}_{4}\right]$ in aqueous and aqueous salt solutions. J. Surfact. Deterg. 22, 327-338 (2019).

44) Trujillo, M.; Schramm, M.P. Measuring critical micelle concentration as a function of cavitand additives using surface tension and dye micellization. Ronald E McNair Postbaccalaureate Achievement Program 14, 155-168(2010).

45) Maiti, K.; Bhattacharya, S.C.; Moulik, S.P.; Panda, A.K. Self-aggregation of synthesized novel bolaforms and their mixtures with sodium dodecylsulfate(SDS) and cetyltrimethylammonium bromide (CTAB) in aqueous medium. J. Phys. Chem. B 122, 867-879 (2010).

46) Barai, M.; Mandal, M.K.; Karak, A.; Bordes, R.; Patra, A.; Dalai, S.; Panda, A.K. Interfacial and aggregation behavior of dicarboxylic amino acid-based surfactants in combination with a cationic surfactant. Langmuir 35, 15306-15314(2019).

47) Patist, A.; Bhagwat, S.; Penfield, A.K.; Aikens, P.; Shah, D.O. On the measurement of critical micelle concentrations of pure and technical-grade nonionic surfactants. J. Surfact. Deterg. 3, 53-58(2000).

48) Gascon, K.N.; Weinstein, S.J.; Antoniades, M.G. Use of simplified surface tension measurements to determine surface excess: an undergraduate experiment. $J$. Chem. Edu. 96, 342-347(2019).

49) Inoue, T.; Ebina, H.; Dong, B.; Zheng, L. Electrical conductivity study on micelle formation of long-chain imidazolium ionic liquids in aqueous solution. J. Colloid Interface Sci. 314, 236-241 (2007).

50) Basu Ray, G.; Chakraborty, I.; Moulik, S.P. Pyrene absorption can be a convenient method for probing critical micellar concentration ( $\mathrm{cmc}$ ) and indexing micellar 
polarity. J. Colloid Interface Sci. 294, 248-54(2006).

51) Sutherland, E.; Mercer, S.M.; Everist, M.; Leaist, D.G. Diffusion in solutions of micelles. What does dynamic light scattering measure? J. Chem. Eng. Data 54, 272-278(2009).

52) Paul, S.; Panda, A.K. Physico-chemical studies on microemulsion: effect of cosurfactant chain length on the phase behavior, formation dynamics, structural parameters and viscosity of water/ (polysorbate- $20+n$-alkanol) $/ n$-heptane water-in-oil microemulsion. J. Surfact. Deterg. 14, 473-486 (2011).

53) Paduszynski, K.; Domanska, U. Viscosity of ionic liquids: an extensive database and a new group contribution model based on a feed-forward artificial neural network. J. Chem. Inform. Mod. 54, 1311-1324 (2014).

54） Maiti, K.; Mitra, D.; Mitra, R.N.; Panda, A.K.; Das, P.K.; Rakshit, A.K.; Moulik, S.P. Self-aggregation of synthesized novel bolaforms and their mixtures with sodium dodecyl sulfate (SDS) and cetyltrimethylammonium bromide (CTAB) in aqueous medium. J. Phys. Chem. B 114, 7499-7508 (2010).

55) Li, J.; Fan, T.; Xu, Y.; Wu, X. Ionic liquids as modulators of physicochemical properties and nanostructures of sodium dodecyl sulfate in aqueous solutions and potential application in pesticide microemulsions. Phys. Chem. Chem. Phys. 18, 29797-29807(2016).

56) Das, C.; Chakraborty, T.; Ghosh, S.; Das, B. Physico- chemistry of mixed micellization: Binary and ternary mixtures of cationic surfactants in aqueous medium. Colloid J. 72, 788-798(2010).

57) Huang, L.; Ren, Z.H. Micellization and interactions for ternary mixtures of amino sulfonate surfactant and nonionic octylphenol polyoxyethylene ethers in aqueous solution: 1 Blending with nonionic surfactants with smaller numbers of hydrophilic unit. J. Mol. Liq. 278, 53-60 (2019).

58) Mahbub, S.; Rub, M.A.; Hoque, M.A.; Khan, M.A.; Kumar, D. Micellization behavior of cationic and anionic surfactant mixtures at different temperatures: Effect of sodium carbonate and sodium phosphate salts. $J$. Phys. Org. Chem. 3967(2019).

59) Mao, J.; Tian, J.; Zhang, W.; Yang, X.; Zhang, H.; Lin, C.; Zhang, Y.; Zhang, Z.; Zhao, J. Effects of a counter-ion salt (potassium chloride) on Gemini cationic surfactants with different spacer lengths. Colloids Surf. A 123619 (2019).

60) Pal, A.; Saini, M. Aggregation behavior of task-specific acidic ionic liquid $N$-methyl-2-pyrrolidinium dihydrogen phosphate $[n m p]\left[\mathrm{H}_{2} \mathrm{PO}_{4}\right]$ in aqueous and aqueous salt solutions. J. Surfact. Deterg. 22, 491-499 (2019).

61) Tan, J.; Shao, J.; Tian, C.; Liao, Z.; Yan, P. Effect of sodium halide on micellar and surface properties of cationic silicone surfactants. J. Surfact. Deterg. 22, 125130 (2019). 